\title{
RTOS Based Image Recognition \& Location Finder Using GPS, GSM and OpenCV
}

\author{
N Papa Rao ${ }^{1}$, G Bhavana ${ }^{2}$, M Lakshmi Ravi Teja ${ }^{3}$ \\ Asst. Professor, Dept. of Electronics \& Communication Engineering at Institute of Aeronautical Engineering ${ }^{1,2,3}$
}

\begin{abstract}
This paper presents the Image Recognition and location finder. With the increasing threat of terrorist, the advanced video surveillance system has to be put into use. The advanced video surveillance system needs to analyze the behaviors of people in order to prevent the occurrence of the potential dangerous case. In recent years, the development of human detection and tracking system has been going forward for several years; many real time systems have been developed. Face based applications such as face recognition and video surveillance systems have been more popular in computer vision during past several years. Such systems perform image processing on the human face and produce throughputs according to their purpose. Face Recognition is a very active research area specializing on how to recognize faces within images or videos. Face recognition compliments Face Detection. Face Detection is the process of finding a 'face' within images or videos and Face Recognition is the process of matching the detected 'face' to one of many the faces known to the file system. There are currently commercially available systems for face recognition, but they are bulky, expensive, and proprietary. Our goal was to create a portable low-cost system using the advanced communication technologies like Global Positioning System (GPS) and GSM (Global System for Mobile communications). An intelligent portable human recognition and identification system is proposed in this project using an ARM 7 based microcontroller and opencv based machine. The system consists of two parts. Unit 1 consists of portable system with BSD Linux including opencv library, usb and serial port to perform the image processing part. Initially using an usb camera interface continuous images are captured and these images are processed with help of Opencv and compared with existing database. If the current images are matching with any of the existing images the system generates command to the unit 2.The unit 2 will perform the location identification using GPS and forward the necessary information about the identified person using GSM/GPRS to concern authorities. This unit contains ARM7 LPC2148 and it is a very popular ARM7 microcontroller with $512 \mathrm{~KB}$ flash, $64 \mathrm{~KB}$ of RAM and with several I/O peripherals, which is more suitable for faster operations. The Keil compiler will be used for building the applications on LPC2148 ARM7 micro controller. Open CV, Linux is used for image processing. Additional sensor units and hardware units are used to build the application.Characters' extraction is developed. They provide a new idea to the vehicle burglarproof area.We proposed that the whole system is built on the platform of embedded system which took advantage of the algorithm of human face recognition. The ARM9-based system combined with the traditional merits of vehicle burglarproof. At the same time, GSM network could be also used in the system. When the crime happened or was about to doing, the messages of alarm would be sent to the vehicle owner as soon as possible. The performance of vehicle burglarproof system has been improved by the system, and the traditional vehicle burglarproof disadvantage could also be solved by this algorithm.
\end{abstract}

Keywords: Video Capture, Human face recognition, ARM7, GPS receiver, GSM, LINUX, OpenCV.

\section{INTRODUCTION}

Generally this face recognition is a big challenge as there is a chance of high uncertainty due to the external lighting conditions, so we are taking the advantage of gray scale images and PCA (Principle Component Analysis), which are less effected to the external environment changes. And mainly a prior step of this face recognition involves face detection which is also a big challenge. For this we are taking the help of pre-designed cascades whose detection of objects is satisfactory.

OpenCV (Open Source Computer Vision Library) which is a library of programming functions mainly aimed at real time computer vision, developed by Intel in 1999 and now supported by Willow Garage. It is free for use under the open source BSD license. The library is cross-platform. It focuses mainly on real-time image processing.

As OpenCV can support all the Image and Signal processing algorithms and which can be ported onto the Linux platform very easily. The major applications of this
OPENCV include 2D \& 3D feature extractions, Ego motion estimation, Facial recognition system, Gesture recognition, Human computer interaction, Mobile robotics, Motion understanding, Object identification, Motion tracking etc.

\section{CHARACTERISTICS OF THE SYSTEM DESIGN}

Intelligent mobile human recognition system integrated a lot of hardware modules such as video capture, GPS positioning and GSM. The design of the system software used the embedded software developing platform based on keil IDE and Linux environment. By the hardware/software co-design, the new intelligent mobile human recognition system implemented.

The main functions are shown as follows:

1) Image capture: When the system works, the camera in the front of the system, collects the data automatically and saves it in the Image buffer. 
2) Human recognition: The system recognizes the human faces by processing the digital images.

3) GPS positioning: The system can correctly send the position by GPS positioning, therefore, the terminals can be coordinated properly.

4) Communication function: then these co-ordinates of the recognized person location can be send to the preloaded mobile number via the GSM network

\section{THE DESIGN OF HARDWARE AND SOFTWARE}

The design of whole system consisted of two part which are hardware and software. The hardware The more details are shown as follows.

\section{A) The design of hardware:}

Intelligent mobile system has composed ARM7 microprocessor peripheral equipment video capture GPS Module wireless telecommunication module SIM300. Furthermore, the modules of LCD, RTC, are connected with the core (LPC2148).

There are several modules consisted of the system as follows:

1)ARM7LPC 2148 : Is suitable for faster application

2)Image Comparison Unit: The image processing unit on OpenCV is PC.

3)LCD: Is used to display the time and place of the detected person

4)RTC: Is used to display the time of detected person.

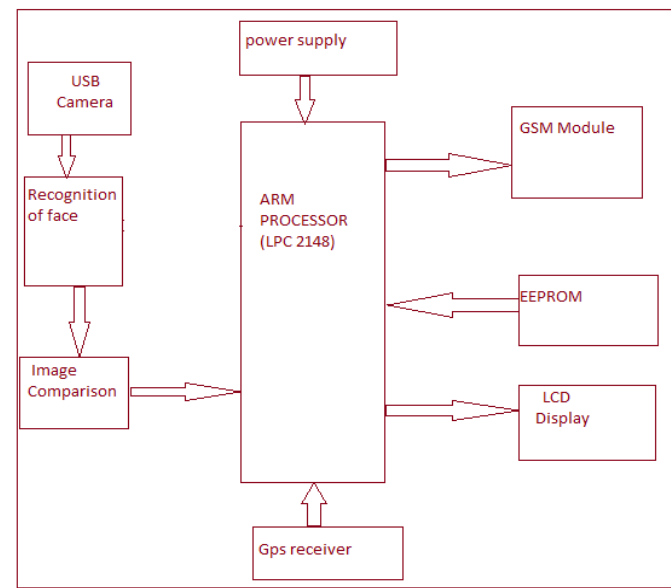

Figure: 1 The Block Diagram of Hardware Structure

\section{B) The design of software:}

The design of software is so vital for the whole system. The design was consisted of two parts included the design of main program flow chart and the algorithm of human face recognition flow chart. This system of software is implemented by the steps as follows: At first, the Boot loader, Linux kernel and the File system are loaded into the PC. In the process of inputting human face, the USB camera, which could fix on the system, will used for acquiring the image of human face. Next step is that the image of human face would be processed by the image processing unit, if the input image matches with the database of images then processor initiates a command to the GPS receiver to get the location and through GSM the system will send the message to the predefined number.

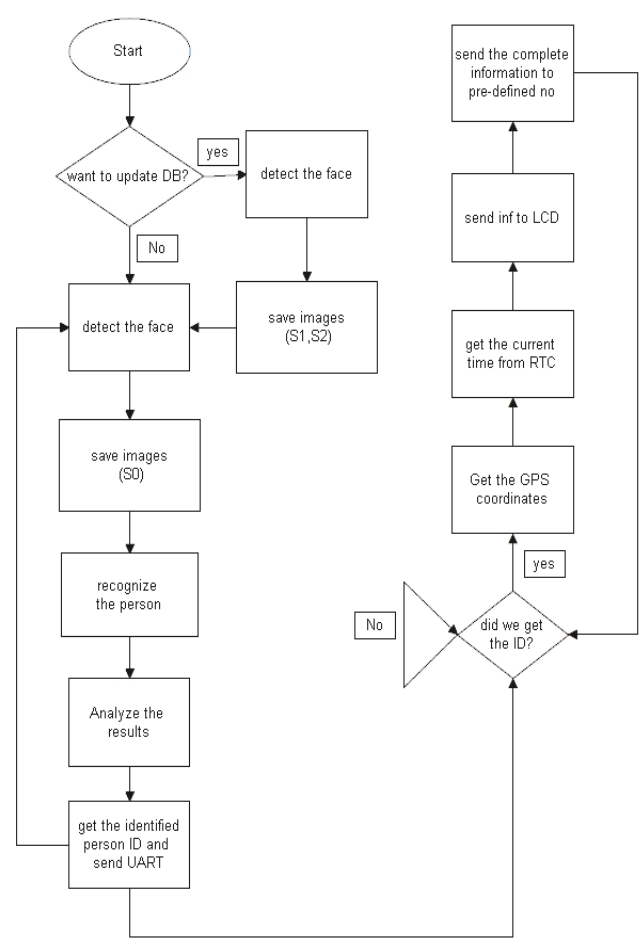

Figure: 2 System Implementation Flow chart

C) The design of human-face recognition algorithm:

The design of algorithm based on human-face recognition was very important for this embedded system. Basically, the process of face recognition follows the face detection first. So as we are designing a basic prototype of human face detection and recognition system, we are taken the cascade classifiers which are already available in the OpenCV library for the face detection and the concept of Eigen values in order to recognition.

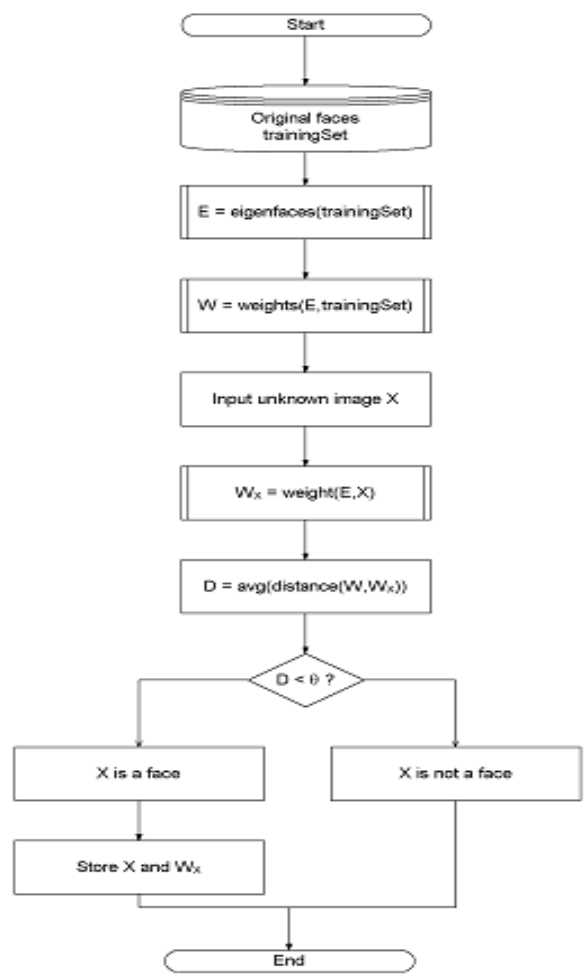

Figure: 3 The detail of flow chart of human-face recognition 
So at first from the data base images, the Eigen values at each and every pixel point is measured and stored in memory. Secondly, from the real time camera the images are taken within short span of time, and the Eigen values at each and every point for these real time images are also found using the same method. At last these two Eigen values are compared with respect to a threshold level, and the further action will be taken.

\section{OPENCV USING FACE RECONGITION}

OpenCV (Open source computer vision) is a library of digital image processing and computer vision, by the Intel Microprocessor Research Lab of the visual interaction group development [9]. OpenCV is written in $\mathrm{C}$ and $\mathrm{C}++$ can be used under the windows system and Linux system, the library is open source. There is active development on interfaces for Python, Ruby, Matlab and other languages. OpenCV was designed for computational efficiency and with a strong focus on real time applications. OpenCV automatically uses the appropriate integrated performance primitives (IPP) library at runtime if that library is installed. The OpenCV library contains over 500 functions that span many areas in vision, including factory product inspection, medical imaging, security, user interface, camera calibration, stereo vision, and robotics. OpenCV provides many standard image processing algorithms, these functions can be directly used in specific video development projects, the scientific research and developers could complete the development of large complex tasks by just adding their own written procedures, it is so-called "get twice the result with half the effort".

\section{SYSTEM SETUP AND DESIGN}

As shown in the fig 4,this system builds a new intelligent human recognition system based on ARM7 embedded processing technology, processing technology of digital videos, image identification technology, GSM wireless mobile telecommunication technology, GPS positioning technique, implements the checking to images continuously taken by a camera. This system has the following features:

1) Image capture: When the system works, the camera collects the data automatically and saves it in the video buffer

2) Human recognition: The system recognizes human face by digital video data

3) Communication function: The human recognition terminal communicates with the mobile phone by the SMS message on the GSM network

4) GPS positioning: The system can correctly send the location coordinates to the hardware module or kit when a person is detected, therefore, the terminals can be coordinated properly.

5) GSM network: The system sends the message to mobile phone via the GSM network wherever the human face is recognized. Intelligent human recognition system is composed of ARM7 microprocessor, peripheral equipment, and video capture device, GPS positioning module, wireless telecommunication module. The detailed hardware composition is shown in fig 1

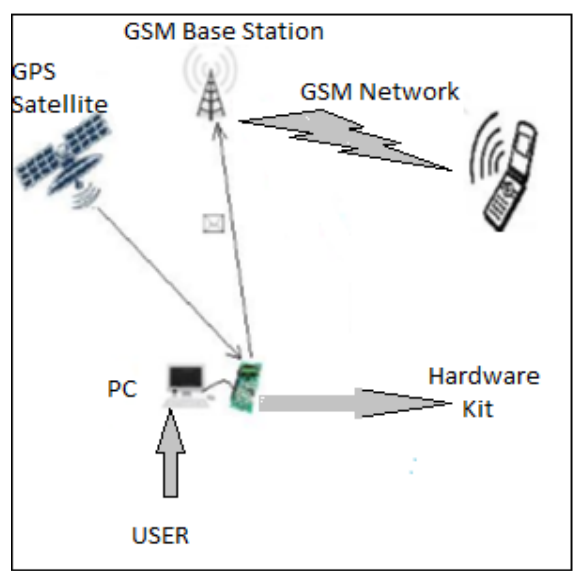

Figure: 4 System Setup

\section{HARDWARE DESIGN}

A. ARM7 microprocessor and peripheral equipment: The circuit of ARM7 microprocessor and peripheral equipment includes an ARM7 chip, a clock circuit, a reset 115 circuit, a 32MB flash memory, a LCD. All of these make up the control and process core of the system.

Fig 1: Hardware structure

B. Video capture:

The video capture module includes: video decoder and output, data buffering and data transmission. The implementation-principle of video capture.The analog video signal captured from the camera is changed to digital signal by SAAA7111A signal-chip video decoder. The SAAA7111A signal chip is initialized and controlled by ARM7. The SAAA7111A output data is written into FIFO buffer. The ARM7 generates interrupt when the data reaches a certain amount. The DMA is started after ARM7 interrupt and sends the video data into buffer.

\section{Equation output and input of GPS data:}

The GPS module can receive the data when connected to ARM7 development-board URAT0 through RS232 port. When the ARM7 chip sends the instruction AT to GPS module, the GPS module starts receiving the data and saves it into memory. This instruction sends the region information with the detected person information to the predefined mobile number.

\section{GSM Wireless Communication:}

The GSM module is installed on the development-board, the RS232 port on the development-board is connected to URAT1 on the ARM7 experiment \& development board in order to implement that the ARM7 chip has control over the GSM communication device. The GSM device is controlled by the ARM7 through AT instructions. The GSM device has message operation instructions and TCP/IP operation instructions. Some simple operations, such as signal strength checking, module vision checking, serial port baud rate checking, SIM card state reading, should be done when the GSM device connects to the support-server center. After making sure that the state which the ARM7experiment- development board connects to the server center by the GSM device is normal, the ARM7 chip outputs the AT instructions in order to send the recognized face information which is acquired by image capture module and image information. 


\section{SOFTWARE DESIGN CYCLE}

The software of the new intelligent human recognition system includes two parts, the OpenCV software and embedded terminal software. The development of the software is based on ADS integrated development environment.

A. Introduction of ADS integrated development environment: The ADS integrated development environment is a microcontroller for ARM which is developed by the ARM Company, its full name is ARM Developer Suit and the mature vision is ADS1.2. ADS1.2 supports the entire ARM microcontroller before ARM10, supports the software debug and JTAG simulate, supports the assembly language, $\mathrm{C}$ and $\mathrm{C}++$ language. It has the merits of high compile efficiency and rich system libraries. The environment can run on Windows98 Windows XP Windows 2000 and Red Hat Linux.

\section{B. OpenCV:}

OpenCV (Open Source Computer Vision) is a library of programming functions mainly aimed at real time computer vision. It is free for use under the open source BSD license. The library is cross-platform. It focuses mainly on real-time image processing. Face detection is a computer technology that determines the locations and sizes of human faces in arbitrary (digital) images. It detects facial features and ignores anything else, such as buildings, trees and bodies.

\section{Navigating the Linux File system}

The Linux filesystem is a tree-like hierarchy hierarchy of directories and files. At the base of the filesystem is the " " " directory, otherwise known as the "root" (not to be confused with the root user). Unlike DOS or Windows filesystems that have multiple "roots", one for each disk drive, the Linux filesystem mounts all disks somewhere underneath the / filesystem. The following table describes many of the most common Linux directories.

The first thing you usually want to do when learning about the Linux filesystem is take some time to look around and see what's there! These next few commands will: a) Tell you where you are, b) take you somewhere else, and c) show you what's there. The following table describes the basic operation of the pwd, cd, and ls commands, and compares them to certain DOS commands that you might already be familiar with.

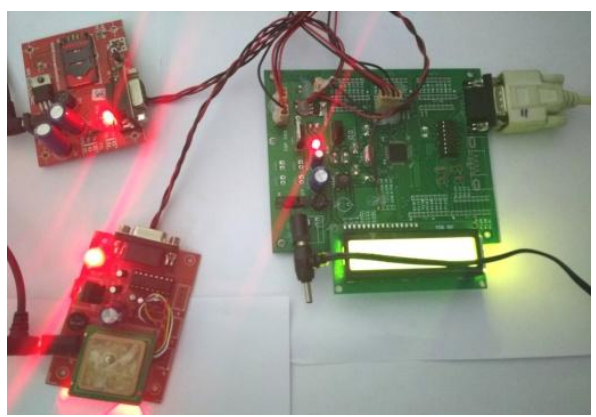

Figure:5 Haredware Design : GPS \& GSM interface with ARM7

Result: The figure 5 below shows complete hardware module, consisting of LPC2148 board, GSM module and
GPS module. The LCD screen on the board shows the longitudinal and latitudinal coordinateThe LCD screen on the board shows the longitudinal and latitudinal coordinate.

\section{CONCLUSION}

As size and portability are the major unique advantages of this OpenCV, it can replace all other image and signal processing tools like MATLAB which is of very huge size and which can't be ported onto any device. The security features were enhanced largely for the stability and liability of human-face recognition. This technology which mentioned in the thesis could be applied to human detection and tracking system.

\section{REFERENCES}

[1] M.S. Bayhan and M.Gokme, "scale and pose invariant real-time face detection and tracking," Proc. The 23rd int. symposium on computer and information science,pp. 1-6, October 2008.

2] G. Yang and T.S. Huang, "Human face detection in complex background," Pattern Recognition, vol.27, no.1, pp.53-63,1994. International Journal of Advanced Trends in Computer Science and Engineering, Vol.2, No.1, Pages : 301 - 304 (2013) Special Issue of ICACSE 2013 - Held on 7-8 January, 2013 in Lords Institute of Engineering and Technology, Hyderabad 304 ISSN 2278-3091

3] C.Kotropoulos and I.Pitas,"Rule - based face detection in frontal views," proc. IEEE Int. On Acoustic, Speech, and signal processing(ICASSP97), vol.4, pp. 2537-2540, April 1997.

[4] E.Osuna, R.freund,and F.Girosi,'Training support vector machines in an application to face detection", proc.IEEE Comp.Soc.Conf.On Computer Vision and pattern Recognition (CVPR 2001), PP.130-136,1997.

[5] H.A.Rowley, S.Baluja,and T.Kanade,"Neural network-based face detection",IEEE Tans. Pattern Analysis is Machine Intelligence, vol.20, issue 1,pp.-38,January 1988.

[6] K.Sung and T.poggio,"Example-based learning for view-based hum- an face detection", IEEE Pattern AnalysisMach.Intelligence, vol.20,issue 1,pp.39-51,January 1998

[7] D.Roth, M.Yang, and N.Ahuja,"A snow based face detector", Advan- ces in Neural Information Processing Systems 12(NIPS12), VOL.12, 2000

[8] P.Viola and M.Jones,"Rapid object detection using a boosted cascade of simple features," Proc.IEEE Computer Vision and pattern Recognition (CVPR 2001) vol.1, pp.1-511-1-518, December 2001.

[9] Ruizhen Liu and shiqi yu, Learning nCV,Beihang University press 2007:247-249.

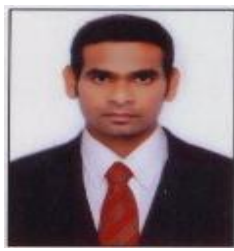

\section{BIOGRAPHIES}

N Papa Rao Working as Asst Professor in the Dept.of ECE at IARE, Hyderabad. 5 years Experience in Teaching and 2 Years in Research. His research interests includes Embedded systems, Image processing, Communications and Wireless sensor networks

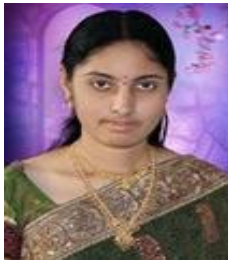

G Bhavana, Working as Asst. Professor in the Dept.of ECE at IARE,Hyderabad.5 years Experience in relative field. She research interests includes Embedded systems, Image processing and Communications

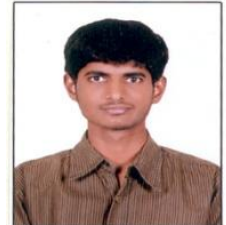

M Lakshmi Ravi Teja, Completed M.Tech in VIT. Working as Asst. Professor in the Dept.of ECE at IARE, Hyderabad. His research interests includes Embedded systems, Sensors 